\title{
SOME TRIGONOMETRIC EXTREMAL PROBLEMS AND DUALITY
}

\author{
SZILÁRD GY. RÉVÉSZ
}

(Received 30 August 1989; revised 19 February 1990)

Communicated by E. N. Dancer

\begin{abstract}
In this paper we present a minimax theorem of infinite dimension. The result contains several earlier duality results for various trigonometrical extremal problems including a problem of Fejér. Also the present duality theorem plays a crucial role in the determination of the exact number of zeros of certain Beurling zeta functions, and hence leads to a considerable generalization of the classical Beurling's Prime Number Theorem. The proof used functional analysis.

1980 Mathematics subject classification (Amer. Math. Soc.) (1985 Revision): primary 42 A 05; secondary 46 B 25.

Keywords and phrases: trigonometrical extremal problems, Borel measures of the $d$-dimensional torus, separation of convex sets, Riesz representation theorem.
\end{abstract}

\section{Introduction}

In [6] Ruzsa proved a duality property between certain extremal quantities. For a different kind of extremal problem a similar duality phenomenon was described in [3]. Later it turned out that the two types of extremal problems are in fact special cases of a class of extremal problems [5]. This class can be parametrized by a continuous variable $r$, where $0 \leq r \leq 1$, and the extremal problems in [3] and [6] belong to the special cases $r=0$ and $r=1$, respectively. So it was natural to look for a more general formulation of duality to cover the general class of extremal problems as well.

This research was partially supported by the Hungarian National Foundation for Scientific Research, grant 1801 .

(C) 1991 Australian Mathematical Society 0263-6115/91 \$A2.00+0.00 
In this paper we present a minimax theorem of several dimensions, which is general enough to cover both the above-mentioned extremal problems and the extremal problem of [2]. Actually, we need to introduce the problem in a several dimensional setting for the sake of [2], where in a certain analytic number theoretical problem this general duality plays a crucial role.

Most of the work presented here is contained in the author's thesis [4]. The author would like to express his gratitude to Professor I. Z. Ruzsa for calling his attention to the paper [6] and for giving useful comments in the course of his work.

\section{The theorem}

Let $d \in \mathbb{N}$ and denote $T^{d}=(\mathbb{R} / 2 \pi \mathbb{Z})^{d}$. We define

(1) $\mathbb{Z}_{+}^{d}:=\left\{\mathbf{z}=\left(z_{1}, \ldots, z_{d}\right) \in \mathbb{Z}^{d}: \exists j<d, z_{1}=\cdots=z_{j}=0, z_{j+1}>0\right\}$.

For any $M, L \subset \mathbb{Z}_{+}^{d}$ we introduce

(2) $\mathscr{F}(M, L):=\left\{f \in \mathscr{T}: f \geq 0, f(x)=1+\sum_{\mathbf{k} \in \mathbf{Z}_{+}^{d}} a(\mathbf{k}) \cos (\mathbf{k} \cdot \mathbf{x})\right.$,

$$
a(\mathbf{k}) \leq 0(\mathbf{k} \notin M), a(\mathbf{k}) \geq 0(\mathbf{k} \notin L)\},
$$

where $\mathscr{T}$ denotes the set of trigonometric polynomials of $d$ variables, and

$$
\mathscr{M}(M, L):=\left\{\begin{array}{r}
\tau \in B M\left(T^{d}\right): d \tau(\mathbf{x}) \sim 2 \sum_{\mathbf{k} \in \mathbf{Z}_{+}^{d}} t(\mathbf{k}) \cos (\mathbf{k} \cdot \mathbf{x}), \\
t(\mathbf{k}) \leq 0(\mathbf{k} \notin M), t(\mathbf{k}) \geq 0(\mathbf{k} \notin L)\},
\end{array}\right.
$$

where $B M\left(T^{d}\right)$ stands for the regular Borel measures of $T^{d}$.

We consider a fixed $\rho \in B M\left(T^{d}\right)$ with Fourier-Lebesgue series

$$
d \rho(\mathbf{x}) \sim 1+2 \sum_{\mathbf{k} \in \mathbf{Z}_{+}^{d}} r(\mathbf{k}) \cos (\mathbf{k} \cdot \mathbf{x}) .
$$

Our goal is to find the extremal quantity

$$
\alpha_{\rho}(M, L):=\inf \{\langle f, \rho\rangle: f \in \mathscr{F}(M, L)\} .
$$


where the scalar product of $f$ and $\rho$ is

$$
\langle f, \rho\rangle=\frac{1}{(2 \pi)^{d}} \int_{T^{d}} f(\mathbf{x}) d \rho(\mathbf{x})=1+\sum_{\mathbf{k} \in \mathbf{Z}_{+}^{d}} a(\mathbf{k}) r(\mathbf{k}) .
$$

Observe that, with the notation

$$
\bar{M}:=\mathbb{Z}_{+}^{d} \backslash M, \quad \bar{L}:=\mathbb{Z}_{+}^{d} \backslash L,
$$

we get

$$
\langle f, \tau\rangle \leq 0 \text { for all } f \in \mathscr{F}(M, L) \text { and } \tau \in \mathscr{M}(\bar{M}, \bar{L}) .
$$

Hence if $\sigma \in B M\left(T^{d}\right)$ satisfies

$$
\sigma-\rho=: \tau \in \mathscr{M}(\bar{M}, \bar{L}),
$$

then

$$
\langle f, \sigma\rangle \leq\langle f, \rho\rangle, \quad f \in \mathscr{F}(M, L),
$$

and hence taking infinum over $f \in \mathscr{F}(M, L)$ we obtain

$$
\alpha_{\sigma}(M, L) \leq \alpha_{\rho}(M, L) \text {. }
$$

On the other hand suppose that $\sigma$ satisfies, for some $t \in \mathbb{R}$, the inequality

$$
\sigma \geq t \lambda \quad\left(d \lambda(\mathbf{x})=d x_{1} d x_{2} \cdots d x_{d}\right) .
$$

Introducing the extremal quantity

$$
\omega_{\rho}(H, K):=\sup \{t: \exists \tau \in \mathscr{M}(H, K), \sigma=\rho+\tau \geq t \lambda\},
$$

we see from (9), (10), (11) and $\langle f, \sigma\rangle \geq\langle f, t \lambda\rangle=t$, that

$$
\alpha_{\rho}(M, L) \geq \omega_{\rho}(\bar{M}, \bar{L}) .
$$

Given this observation our aim is to prove the sharpness of (13).

TheOREM. Let $M, L \subset \mathbb{Z}_{+}^{d}$ and $\rho \in B M\left(T^{d}\right)$ be arbitrary. We have

$$
\alpha_{\rho}(M, L)=\omega_{\rho}(\bar{M}, \bar{L}) \text {. }
$$

\section{Proof of the duality theorem}

Since $\rho, M, L$ and $\bar{M}, \bar{L}$ are fixed once and for all, we use $\alpha, \mathscr{F}$ and $\omega, \mathscr{M}$ without writing out $\rho, M, L$ and $\rho, \bar{M}, \bar{L}$ respectively. In view of (13) it is enough to prove $\omega \geq \alpha$.

We can suppose $\alpha>-\infty$ and as $f \equiv 1 \in \mathscr{F}$, we have $\alpha \leq 1$. For any $\mathbf{m} \in M$ and $\mathbf{l} \in L, 1+\cos (\mathbf{m} \cdot \mathbf{x}) \in \mathscr{F}$ and $1-\cos (\mathbf{l} \cdot \mathbf{x}) \in \mathscr{F}$. Now if $\alpha=1$ 
then $1=\alpha \leq\langle 1+\cos (\mathbf{m} \cdot \mathbf{x}), \rho\rangle=1+r(\mathbf{m})$ and $1=\alpha \leq\langle 1-\cos (\mathbf{l} \cdot \mathbf{x}), \rho\rangle=$ $1-r(\mathbf{l})$, hence $r(\mathbf{m}) \geq 0 \quad(\mathbf{m} \in M), r(\mathbf{l}) \leq 0 \quad(\mathbf{l} \in L)$ and so $\tau:=\lambda-\rho \in \mathscr{M}$, and so $\sigma=\rho+\tau=\lambda$ can occur in (12) and $\omega=1$.

Therefore, we can suppose $-\infty<\alpha<1$. Moreover, we can suppose $\alpha=0$, since for any $\alpha \in(-\infty, 1)$ we can consider

$$
\rho^{*}=\frac{1}{1-\alpha}(-\alpha \cdot \lambda+\rho), \quad \alpha^{*}=\alpha_{\rho^{*}}(M, L), \quad \omega^{*}=\omega_{\rho^{*}}(\bar{M}, \bar{L})
$$

and trivially

$$
\alpha^{*}=\frac{-\alpha}{1-\alpha}+\frac{1}{1-\alpha} \alpha=0, \quad \omega^{*}=\frac{-\alpha}{1-\alpha}+\frac{1}{1-\alpha} \omega
$$

proves $\alpha=\omega$ if $\omega^{*}=0$. Hence we take now $\alpha=0$ and prove $\omega \geq 0$.

Denote

$$
\mathscr{P}:=\left\{h \in C\left(T^{d}\right): h>0\right\},
$$

and with conditions on the coefficients identical to $\mathscr{F}$, introduce

$$
\begin{aligned}
& \mathscr{G}:=\left\{g \in \mathscr{T}:\langle g, \rho\rangle=0, g(\mathbf{x})=1+\sum_{\mathbf{n} \in \mathbf{Z}_{+}^{d}} a(\mathbf{n}) \cos (\mathbf{n} \cdot \mathbf{x}),\right. \\
& a(\mathbf{n}) \leq 0(\mathbf{n} \notin M), a(\mathbf{n}) \geq 0(\mathbf{n} \notin L)\} .
\end{aligned}
$$

In the Banach space $C\left(T^{d}\right)$, where the norm is the supremum norm as usual, $\mathscr{P}$ forms an open, nonvoid convex cone, and $\mathscr{G}$ is another convex set. Then $\mathscr{G}$ is nonvoid since for any $f_{0} \in \mathscr{F}$ with $0 \leq\left\langle f_{0}, \rho\right\rangle<1$ (such $f_{0}$ must exist since $\alpha=0<1$ ) we have

$$
g_{0}(\mathbf{x}):=\frac{1}{1-\langle f, \rho\rangle}(f-\langle f, \rho\rangle) \in \mathscr{G} .
$$

Moreover, $\mathscr{P} \cap \mathscr{G}=\varnothing$. Indeed, for any $g \in \mathscr{P} \cap \mathscr{G}, 0<\min g \leq 1$ and so with $\delta=\frac{1}{2} \min g$ we have

$$
F:=\frac{g-\delta}{1-\delta} \in \mathscr{F},
$$

and hence $0=\alpha \leq\langle F, \rho\rangle=-\delta /(1-\delta)<0$, a contradiction. Therefore we can apply the separation theorem of convex sets (cf. [1, Corollary 2.2.2]) to $\mathscr{P}$ and $\mathscr{G}$, which furnishes a nontrivial continuous linear functional $I$ satisfying

$$
I \mathscr{P} \geq 0 \geq I \mathscr{G}, \quad I 1=1 .
$$


Here $I 1=1$ is a matter of normalization since $I \mathscr{P} \geq 0$ and $I 1=0$ would imply $I \mathscr{P}=0$ and hence $I=0$, and therefore $I 1>0$ is guaranteed. The separation constant can be chosen to be 0 since $\mathscr{P}$ is a cone and $I \mathscr{P} \neq 0$ implies $I \mathscr{P}=(0, \infty)$ or $[0, \infty)$ once $I \mathscr{P}$ is bounded from below. Also, we can suppose that $I$ is even in the sense that

$$
I(f(\mathbf{x}))=\frac{1}{2^{d}} \sum_{\varepsilon_{1}, \ldots, \varepsilon_{d}= \pm 1} I\left(f\left(\varepsilon_{1} x_{1}+\cdots+\varepsilon_{d} x_{d}\right)\right)
$$

since we can define a new functional by the right hand side of (17) if it does not hold for $I$ itself. Applying the representation theorem of F. Riesz (cf. [1, Theorem 4.10.1]) we obtain a $\mu \in B M\left(T^{d}\right)$ which satisfies, according to (16) and (17),

$$
I=\frac{1}{(2 \pi)^{d}} \int_{T^{d}} \cdot d \mu, \quad d \mu(\mathbf{x}) \sim 1+\sum_{\mathbf{k} \in \mathbf{Z}_{+}^{d}} b(\mathbf{k}) \cos (\mathbf{k} \cdot \mathbf{x}) .
$$

We define the index sets

$$
N_{+}=\left\{\mathbf{k} \in \mathbb{Z}_{+}^{d}: r(\mathbf{k}) \gtreqless 0\right\}, \quad M_{+}=\underset{0}{N_{+}} \cap M, \quad L_{+}=L \cap \underline{\underline{0}} \underline{N_{+}},
$$

where the three alternatives $(+, 0,-)$ are to be understood separately. Accordingly, we denote the elements of $M_{+}$by $\mathbf{m}_{+}$, elements of $M_{0}$ by $\mathbf{m}_{0}$, etc. For any $g_{0} \in \mathscr{G}$ and $a>0$

$$
g_{0}+a \cos \left(\mathbf{m}_{0} \cdot \mathbf{x}\right) \in \mathscr{G}, g_{0}-a \cos \left(\mathbf{l}_{0} \cdot \mathbf{x}\right) \in \mathscr{G},
$$

and hence (16) and (18) give after $a \rightarrow+\infty$ the inequalities

$$
b\left(\mathbf{m}_{0}\right) \leq 0, \quad b\left(\mathbf{l}_{0}\right) \geq 0 .
$$

Suppose now that $\mathbf{n} \in M_{-} \cup L_{+}$. Clearly,

$$
g_{\mathbf{n}}(\mathbf{x}):=1-\frac{1}{r(\mathbf{n})} \cos (\mathbf{n} \cdot \mathbf{x}) \in \mathscr{G},
$$

and so (16) and (18) now yield $1 \leq b(\mathbf{n}) / r(\mathbf{n})$ and

$$
s:=\inf \left\{\frac{b(\mathbf{n})}{r(\mathbf{n})}: \mathbf{n} \in M_{-} \cup L_{+}\right\} \geq 1 .
$$

Finally we define for $\mathbf{k}, \mathbf{n} \in N_{+} \cup N_{-}$the function

$$
f_{\mathbf{k}, \mathbf{n}}(\mathbf{x}):=\frac{1}{r(\mathbf{k})} \cos (\mathbf{k} \cdot \mathbf{x})-\frac{1}{r(\mathbf{n})} \cos (\mathbf{n} \cdot \mathbf{x}) \text {. }
$$

Then $\left\langle f_{\mathbf{k}, \mathbf{n}}, \rho\right\rangle=0$ and for a fixed $g_{0} \in \mathscr{G}$ and $a>0$ we have

$$
\begin{array}{ll}
g_{0}-a f_{\mathbf{k}, \mathbf{n}} \in \mathscr{G} & \left(\mathbf{k} \in L_{+}, \mathbf{n} \in L_{-}\right), \\
g_{0}+a f_{\mathbf{k}, \mathbf{n}} \in \mathscr{G} & \left(\mathbf{k} \in M_{+}, \mathbf{n} \in M_{-}\right), \\
g_{0}+a f_{\mathbf{k}, \mathbf{n}} \in \mathscr{G} & \left(\mathbf{k} \in M_{+}, \mathbf{n} \in L_{+}\right), \\
g_{0}-a f_{\mathbf{k}, \mathbf{n}} \in \mathscr{G} & \left(\mathbf{k} \in M_{-}, \mathbf{n} \in L_{-}\right) .
\end{array}
$$


Again, we refer to (16), IG $\leq 0$ and (18) to obtain after $a \rightarrow+\infty$ the inequalities

$$
\begin{aligned}
& \frac{b\left(\mathbf{l}_{+}\right)}{r\left(\mathbf{l}_{+}\right)} \geq \frac{b\left(\mathbf{l}_{-}\right)}{r\left(\mathbf{I}_{-}\right)}, \quad \frac{b\left(\mathbf{m}_{-}\right)}{r\left(\mathbf{m}_{-}\right)} \geq \frac{b\left(\mathbf{m}_{+}\right)}{r\left(\mathbf{m}_{+}\right)}, \\
& \frac{b\left(\mathbf{l}_{+}\right)}{r\left(\mathbf{I}_{+}\right)} \geq \frac{b\left(\mathbf{m}_{+}\right)}{r\left(\mathbf{m}_{+}\right)}, \quad \frac{b\left(\mathbf{m}_{-}\right)}{r\left(\mathbf{m}_{-}\right)} \geq \frac{b\left(\mathbf{l}_{-}\right)}{r\left(\mathbf{I}_{-}\right)} .
\end{aligned}
$$

Comparing (23) and (21) we obtain that for a certain real $s \geq 1$

$$
\frac{b(\mathbf{n})}{r(\mathbf{n})} \geq s \geq \frac{b(\mathbf{k})}{r(\mathbf{k})} \quad\left(\mathbf{n} \in M_{-} \cup L_{+}, \mathbf{k} \in M_{+} \cup L_{-}\right) .
$$

Now let us define

$$
\tau:=\left(1-\frac{1}{s}\right) \lambda+\frac{1}{s} \mu-\rho, \quad d \tau(\mathbf{x}) \sim 2 \sum_{\mathbf{k} \in \mathbf{Z}_{+}^{d}} t(\mathbf{k}) \cos (\mathbf{k} \cdot \mathbf{x}) .
$$

Then it is easy to check that the constant term in the Fourier-Lebesgue series of $\tau$ is zero, and (24) along with (20) can be expressed as

$$
t(\mathbf{m}) \leq 0 \quad(\mathbf{m} \in M), \quad t(\mathbf{l}) \geq 0 \quad(\mathbf{l} \in L)
$$

whence

$$
\tau \in \mathscr{M}
$$

Now with $t=1-1 / s \geq 0$ we infer from $\mu \geq 0$ and (25)-(27) that $\sigma:=$ $\tau+\rho \geq t \lambda$ and so $\omega \geq t \geq 0$, which completes the proof of our theorem.

\section{References}

[1] R. E. Edwards, Functional analysis, (Holt-Rinehart-Winston, New York, Toronto, London, 1965).

[2] Sz. Gy. Révész, 'On Beurling's prime number theorem', preprint, Mathematical Institute of the Hungarian Academy of Sciences, No. 5, 1990.

[3] Sz. Gy. Révész, 'Extremal problems and a duality phenomenon', Proceedings of the IMACS conference held in Dalian, China, 1989, edited by Alan G. Law, to appear.

[4] Sz. Gy. Révész, Polynomial extremal problems (in Hungarian), (thesis for the "candidate degree", 1988, Budapest).

[5] Sz. Gy. Révész, 'On a class of extremal problems', Journal of Approx. Th. and Appl., to appear. 
[6] I. Z. Ruzsa, 'Connections between the uniform distribution of a sequence and its differences', Topics in classical number theory, Coll. Math. Soc. J. Bolyai 34, edited by P. Erdös and G. Halász, pp. 1419-1443 (North-Holland, Amsterdam, New York, Budapest, 1981).

Mathematical Institute

Hungarian Academy of Sciences

Budapest, POB 127, 1364

Hungary 